\title{
Study of individual pulses at the Antarctic high-altitude neutron monitor DOMC
}

\author{
Markus Similä , Stepan Poluianov ${ }^{(\mathbb{C})}$, Ilya Usoskin ${ }^{(1)}$ \\ Correspondence \\ Sodankylä Geophysical Observatory, University of Oulu, Finland \\ markus.simila01@gmail.com, stepan.poluianov@oulu.fi, ilya.usoskin@oulu.fi
}

\section{OPEN ACCESS}

This work is published under the Creative Commons Attribution 4.0 International licence (CC BY 4.0) Please note that individual, appropriately marked parts of the work may be excluded from the licence may be excluded from the licence other copyright conditions. other copyright conditions.
If such thirdparty material is not If such thirdparty material is not under the Creative Commons license, any copying, editing or public reproduction is only permitted with the prior consent of the respective copy right owner or on the basis of relevan legal authorization regulations.

\section{Keywords}

neutron monitor; atmospheric cascade; cosmic rays

\begin{abstract}
A pair of neutron monitors (NMs) is installed on the high Central Antarctic plateau, at the Concordia station (3200 m altitude) and measures the nucleonic component of nucleonic-muon-electromagnetic cascades induced by high-energy cosmic rays in the atmosphere. The installation includes two NMs: DOMC, a standard mini-NM, and a bare (lead-free) DOMB NM. The newly installed data acquisition (DAQ) system records individual pulses corresponding to mostly neutrons in the detector's counting tube. Here we analyze different types of pulses and study the distribution of the waiting times between individual pulses as well as the pulse height, recorded by the DOMC NM during a quiet period of January 2020. The distribution appears doublepeaked with peaks corresponding to the frequency of individual atmospheric cascades and the intra-cascade variability, respectively. We discuss also the nature of different components contributing to the pulses and separation of the signal from noise. It is shown that the waiting-time distribution has distinguished timescales, $>30$ ms defined by the cosmic-ray induced atmospheric cascades, and $<10$ ms reflecting the intra-cascade variability. The new DAQ system allows one to study the development of the atmospheric cascade.
\end{abstract}

\section{Introduction}

A neutron monitor (NM) is a proportional counter designed to measure the nucleonic component of complex cascades induced by energetic cosmic-ray particles in the atmosphere (Moraal et al. 2000; Simpson 2000). NMs of the standard design count the number of pulses on the central wire per unit time (count rate). The DOMC NM is a mini-NM built following the standard NM design, viz. including two layers of moderator and a lead shield (Poluianov et al. 2015). It is located on the Central Antarctic plateau at the Franco-Italian Concordia station (3200 m altitude) and has the world's highest access for low-energy cosmic-ray particles on the ground. Since late 2019, a new data acquisition system (DAQ) operates on the DOMC NM, digitizing each pulse with the 2-MHz sampling frequency (Strauss et al., Poluianov, Usoskin \& Strauss, this issue). Most of the pulses have a $0.2-2.5 \mathrm{~V}$ height and $10-40 \mu$ s duration. The latter corresponds to a typical NM dead time. Here we provide results of the first analysis of the waiting-time distribution of individual pulses for DOMC NM, for a very quiet period (1-27 January 2020), in relation to the development of the nucleonic cascade in the atmosphere. 


\section{Pulse analysis}

In total, about 60 millions of individual pulses have been recorded by the new DAQ system of DOMC NM during January 2020. The distribution of the amplitude and duration of pulses is shown in figure 1. Four different branches can be observed: A - the main branch, where each pulse has a well-defined shape and can be clearly separated from the others (figure $2 \mathrm{~A}$ ) so that pulse drops below the detection threshold before the new pulse starts; $\mathrm{B}$ - noise dominated branch, where pulses are low, short and not shaped (figure 2B); C - multiple (typically double) well-defined peaks where the second one starts before the first one drops below the threshold (figure 2C); D - multiple (can be numerous) high-amplitude peaks following with very short (10-30 $\mu \mathrm{s})$ time separation (figure 2D), we argue that it is likely to be caused by multiple intra-counter avalanches. About $93 \%$ of all pulses lies in the branches A and C, branch B comprises 7\%, and D accounts for only $0.05 \%$ of all pulses. We note that increasing the detection threshold to $0.5-0.6 \mathrm{~V}$ reduces the noise (branch $\mathrm{B}$ ) by an order of magnitude. In the following, we have performed an analysis of the data by applying a threshold of $0.5 \mathrm{~V}$, so that the contribution of unwanted branches $\mathrm{B}$ and $\mathrm{D}$ is reduced to $<1 \%$.

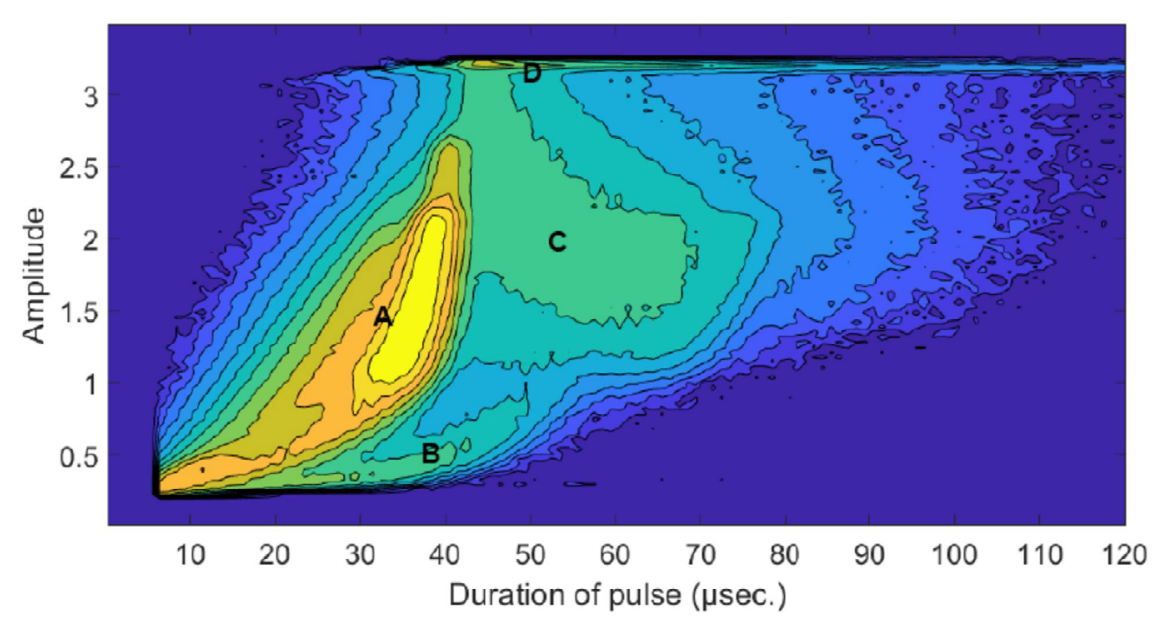

Figure 1: Amplitude (in volts) versus duration (in microseconds) log-distribution of $~ 60$ millions of pulses recorded by DOMC NM in January 2020. The detection threshold is set for $0.2 \mathrm{~V}$. Four main branches are indicated: $A$ - the main branch where well-shaped pulses can be clearly separated; B - noise-dominated region; C - multiple pulses originated from the atmospheric cascades; D - intra-detector cascades. Examples of pulse's time profiles are shown in figure 2.

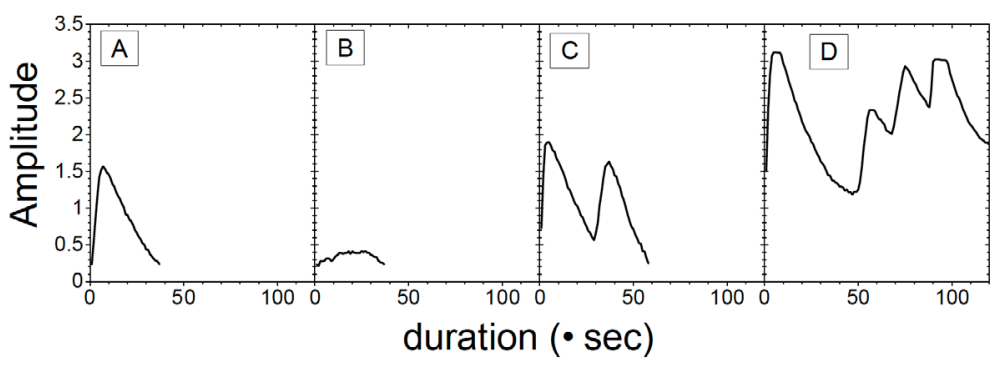

Figure 2: Typical pulse profiles (amplitude in volts vs time in microseconds) for the four branches A-D in figure 1 (as indicated by the panels). 
Next, we computed the waiting time, defined as the time interval between onsets (zero-times in figure 2) of subsequent pulses. The distribution (weighted with the bin width for better visibility) of the waiting times is shown in figure 3. One can see that it has two distinct scales. One peak in the distribution corresponds to $\sim 2 \mathrm{~ms}(0.3-10 \mathrm{~ms})$ and is close to the log-normal shape. The other peak corresponds to the waiting times greater than $\sim 30 \mathrm{~ms}$ followed by a purely exponential tail corresponding to the mean count rate $(\sim 20 \mathrm{~Hz})$ of the detector. Smooth behaviour is observed in between. Here we note that the second peak corresponds to the expected standard distribution for cosmic-ray induced atmospheric cascades.

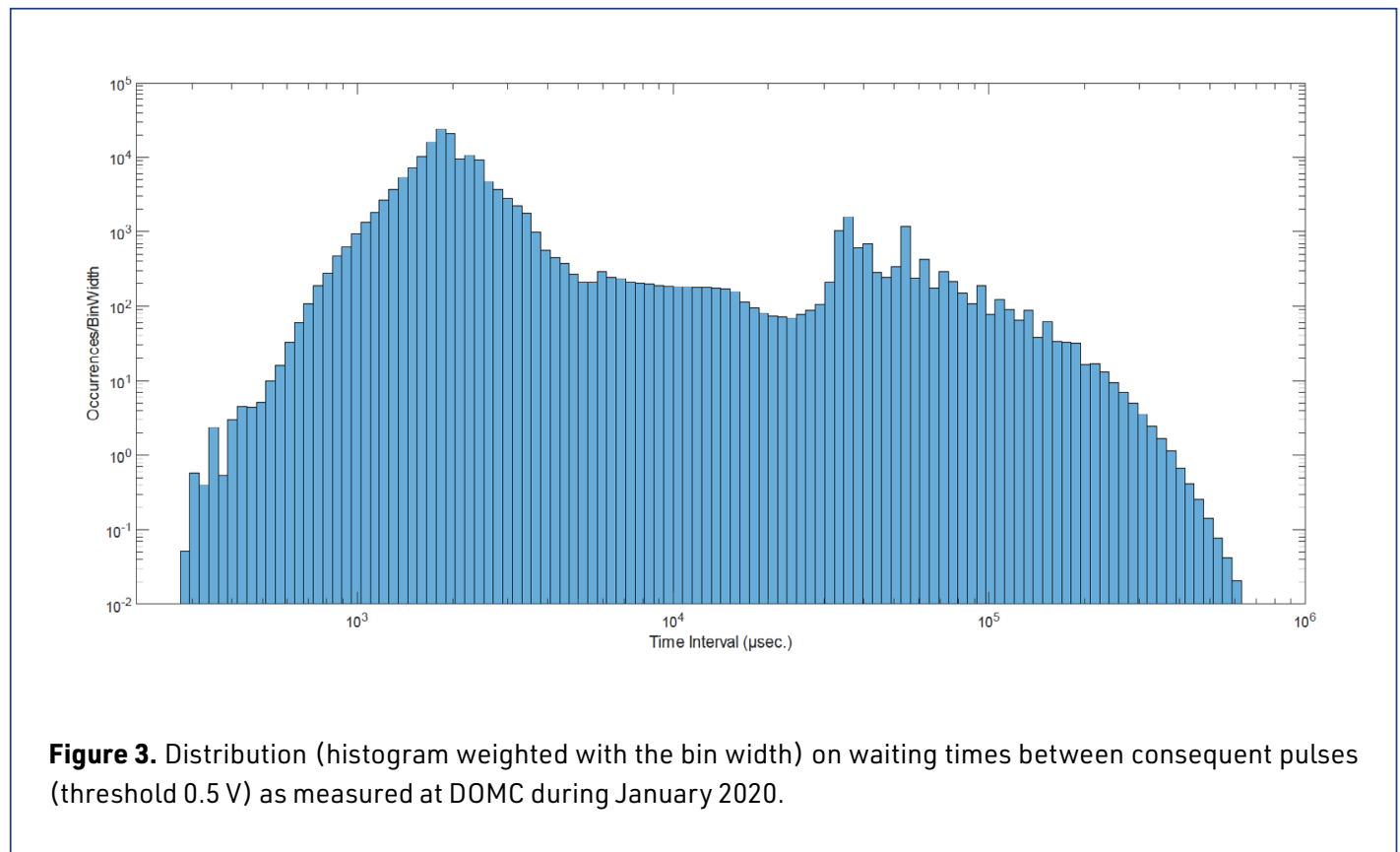

The waiting time is defined by the waiting time between individual cascades caused by different cosmic-ray primary particles and reflects the behaviour of the cosmic-ray flux impinging on the Earth's atmosphere. The second peak dominates the count rate of the NM (because of the weighting, it looks smaller than the first one). The first peak with smaller waiting times corresponds to the intra-cascade multiplicity, viz. multiple secondary particles arriving from the atmospheric cascade induced by the same primary particles and mostly reflects the development of the cascade in the atmosphere. We note that, because of the high-altitude location of the DOMC NM, the first intra-cascade peak may be enhanced, but it may be smaller at sea level but more intense at higher cutoffs (lower latitudes). By analysis of individual pulses, one can study details of the atmospheric transport of cosmic rays.

\section{Discussion and conclusion}

We have analyzed the amplitude and waiting-time distribution of $\sim 60$ millions of individual pulses digitized by DAQ of DOMC NM during a quiet period of January 2020. We have shown that the raw data contain several types of pulses: single and multiple pulses from the atmospheric cascade ( $~ 93 \%$ of all pulses), multiple pulses from the intra-detector avalanches $(<0.1 \%)$, and low-amplitude noise $(\sim 7 \%)$. Noise can be significantly reduced by increasing the discriminator threshold from the built-in $0.2 \mathrm{~V}$ to $0.5 \mathrm{~V}$. Two distinct peaks can be clearly identified in the waiting-time distribution of pulses: one, with a purely exponential tail, lies in the time range of longer than $30 \mathrm{~ms}$ reflecting the frequency of individual atmospheric cascades and corresponding to the mean count 
rate of DOMC NM ( $\sim 20 \mathrm{~Hz}$ ); the other, with the time range of 1-10 ms and a nearly log-normal distribution, corresponds to the variability of secondary particles within the same atmospheric cascade, viz. intra-cascade variability. Similar results were obtained for different values of the discriminator threshold, ranging from $0.2-2.0 \mathrm{~V}$.

The proposed method can clearly distinguish between intra- and inter-cascade variability and can be used to study multiplicities and details of the development of the nucleonic cascades induced by energetic particles in the atmosphere.

\section{Acknowledgments}

The work was partly supported by FINNARP and Academy of Finland (projects 264378 CRIPA and 304435 CRIPA-X).

\section{References}

Moraal, H., Belov, A, Clem, J., 2000, Space Sci. Rev., 93, 285-303, D0I: https://dx.doi.org/10.1023/A:1026504814360

Poluianov, S., Usoskin, I., Mishev, A., et al., 2015. J. Astron. Space Sci., 32, 281, 2015, DOI: https://dx.doi.org/10.5140/JASS.2015.32.4.281

Simpson, J.A., 2000, Space Sci. Rev. 93, 11-32, D0I: https://dx.doi.org/10.1023/A:1026567706183

Strauss, D.T., Poluianov, S., van der Merwe, C., et al., J. Space Weather Space Clim., 10, 39, 2020, D0I: https://dx.doi.org/10.1051/ swsc/2020038 